\title{
Spin Seebeck effect in an (In,Ga)As quantum well with equal Rashba and Dresselhaus spin-orbit couplings
}

\author{
Jeremy Capps and D. C. Marinescu \\ Department of Physics and Astronomy, Clemson University, Clemson, South Carolina 29634, USA \\ Andrei Manolescu \\ University of Rejkyavik, Iceland \\ (Received 24 March 2015; revised manuscript received 21 January 2016; published 9 February 2016)
}

\begin{abstract}
We demonstrate that a spin-dependent Seebeck effect can be detected in quantum wells with zinc-blend structure with equal Rashba-Dresselhaus spin-orbit couplings. This theory is based on the establishment of an itinerant antiferromagnetic state, a low total-energy configuration realized in the presence of the Coulomb interaction enabled by the $\mathbf{k}=0$ degeneracy of the opposite-spin single-particle energy spectra. Transport in this state is modeled by using the solutions of a Boltzmann equation obtained within the relaxation time approximation. Numerical estimates performed for realistic GaAs samples indicate that at low temperatures, the amplitude of the spin Seebeck coefficient can be increased by scattering on magnetic impurities.
\end{abstract}

DOI: 10.1103/PhysRevB.93.085307

\section{INTRODUCTION}

Of all the rich and varied phenomenology associated with the presence of the Rashba and Dresselhaus spin-orbit interactions in quantum wells with zinc-blend structure, the properties of the regime in which the two coupling strengths are equal have attracted considerable attention since they showcase unusual features such as very long spin relaxation rates [1-3] or the absence of the antilocalization correction to the conductivity $[4,5]$. In this paper we discuss a possible add-on to this panoply, namely the existence of a spin Seebeck effect.

The idea of incorporating spin effects in the usual chargeonly thermoelectric phenomenology, a general topic known as spin caloritronics [6,7], has been recently motivating a significant effort in theoretical and experimental research. The experimental detection of a spin-dependent current driven by a temperature gradient in ferromagnets [8,9], ferromagnetic semiconductors [10], and semiconductors in the presence of a magnetic field [11] helped define the spin Seebeck effect as a fundamental property of physical systems characterized by an intrinsic spin polarization. The original picture of the spin-dependent thermal current originating from unequal populations of spins has evolved to incorporate transport through spin waves [12], once the spin Seebeck effect was observed in magnetic insulators [13].

Defined as the proportionality coefficient between the electric field that appears in an open circuit and the thermal gradient that induces it, the Seebeck coefficient or thermopower $S$ was early on recognized as a measure of the efficiency of transport. In normal metallic systems its numerical value is small, as it is determined by the ratio of the thermal energy to the Fermi energy. This result is a consequence of the almost complete cancellation of the energy current carried by electrons above the Fermi level with that carried by electrons below the Fermi level.

Disrupting this cancellation requires the presence of two factors, as it was recognized long ago in Ref. [14], where the usual charge Seebeck coefficient was calculated in a system with a spin-density-wave ground state. First, it is necessary that two distinct, unequal groups of electrons exist, such as one can obtain through spin polarization. Second, a scattering mechanism that provides a unidirectional energy transfer between these groups, needs to be introduced, for example one that involves spin flips. While the latter factor can be controlled rather easily through doping with magnetic impurities, it is the first criterion that drastically limits the number of systems that can be susceptible to this approach, as naturally created and sustained spin polarization is rare.

In this paper we generalize these principles to the case of spin Seebeck effect and discuss how both these criteria are fulfilled in a quantum well with spin-orbit interaction in the regime where the strengths of the Rashba and Dresselhaus couplings are equal, leading as a consequence to an enhanced spin Seebeck coefficient. Central to the argument presented here is the existence of an antiferromagnetic ground state of the electron system in the well when $\alpha$, the strength of the Rashba coupling, is equal to $\beta$, the strength of the linear Dresselhaus coupling, a configuration that results from the minimization of the total energy of the system in the presence of the Coulomb interaction $[15,16]$. Since the spin Seebeck effect is directly conditioned by the antiferromagnetic spin alignment, otherwise being absent in a paramagnetic state, its detection in a quantum well prepared in the $\alpha=\beta$ regime can serve as an experimental probe for the existence of such a long range magnetic order. Furthermore, we follow Ref. [14] and show that the magnitude of the Seebeck coefficient can be greatly enhanced at low temperatures by scattering on magnetic impurities since this process introduces an energy-dependent lifetime which increases the difference between the energy currents carried by the opposite-spin electrons. Our theoretical model predicts this phenomenology to occur in an independent, single quantum well with SOI, a different setup than the recent analysis of the spin-dependent transport in a quantum well with SOI connected to ferromagnetic reservoirs [17]. Moreover, the itinerant antiferromagnetic state in which the spin Seebeck effect can be measured represents an interesting alternative to the spin wave model for temperature induced spin currents in ferromagnetic insulators [12]. Because in our problem the spin polarization is related to a fundamental property of the ground state, its coherence is maintained over large distances, making 
this system one of the most attractive candidates for exploring spin-dependent thermoelectric effects.

In the first section of this paper we outline the theory of the formation of the weak itinerant antiferromagnetic ground state in a quantum well with equal Rashba-Dresselhaus spin-orbit interactions $[15,16]$. In essence, in the presence of the Coulomb interaction, the degeneracy in the opposite-spin single-particle states that is realized at $\mathbf{k}=0$ enables a lowering of the total energy of the many-body state when the electron spin is allowed to freely rotate in the momentum state. The quasiparticles thus created are linear combinations of single particle states with spin up and down whose polarization changes continuously in $\mathbf{k}$ space. This antiferromagnetic spin alignment leads to a nonzero value of the volumic spin polarization. The transport theory of these quasiparticles formulated within the time relaxation approximation of the Boltzmann equation is presented in the second section. Finally, we calculate the spin thermopower in a realistic system and present its temperature variation as a function of the strength of the scattering on magnetic impurities.

\section{THE DESCRIPTION OF THE SYSTEM}

We consider a two-dimensional electron system in the $(x, z)$ plane (the $\hat{y}$ axis is perpendicular on the plane) that experiences a Rashba and Dresselhaus spin-orbit interaction linear in the electron momentum, of strengths $\alpha$ and $\beta$, respectively. The single particle Hamiltonian of an electron of wave-vector $\mathbf{k}=\left\{k_{x}, k_{z}\right\}$ and $\operatorname{spin} \sigma=\left\{\sigma_{x}, \sigma_{y}, \sigma_{z}\right\}$ is given by

$$
H_{1}=\frac{\hbar^{2} k^{2}}{2 m^{*}}+\hbar \alpha\left(\sigma_{z} k_{x}-\sigma_{x} k_{z}\right)+\hbar \beta\left(\sigma_{z} k_{z}-\sigma_{x} k_{x}\right) .
$$

A real space rotation (clockwise about $\hat{y}$ ) in the $x-z$ plane is used to introduce the new momentum coordinates $k_{x}^{\prime}=\left(k_{x}+\right.$ $\left.k_{z}\right) / \sqrt{2}$ and $k_{z}^{\prime}=\left(k_{z}-k_{x}\right) / \sqrt{2}$ and the new spin projections $\sigma_{x}^{\prime}=\left(\sigma_{x}+\sigma_{z}\right) / \sqrt{2}$ and $\sigma_{z}^{\prime}=\left(\sigma_{z}-\sigma_{x}\right) / \sqrt{2}$. In this frame, the expression of the Hamiltonian becomes

$$
H_{1}=\frac{\hbar^{2}\left(k_{x}^{\prime}\right)^{2}}{2 m^{*}}+\frac{\hbar^{2}\left(k_{z}^{\prime}\right)^{2}}{2 m^{*}}+\hbar(\alpha+\beta) \sigma_{z}^{\prime} k_{x}^{\prime}-\hbar(\alpha-\beta) \sigma_{x}^{\prime} k_{z}^{\prime} .
$$

When $\alpha= \pm \beta$, one of the two spin-orbit terms cancels and the Hamiltonian commutes with the remaining component of the spin operator. We select $\alpha=\beta$, drop the prime indices, and rewrite the Hamiltonian in a simplified form,

$$
H_{1}=\frac{\hbar^{2}}{2 m^{*}}\left(k_{x}-Q \sigma_{z}\right)^{2}+\frac{\hbar^{2} k_{z}^{2}}{2 m^{*}}-\frac{\hbar^{2} Q^{2}}{2 m^{*}},
$$

where $\mathbf{Q}=2 m^{*} \alpha / \hbar \hat{x}$ is a displacement vector in the momentum space parallel to the $\hat{x}$ axis.

For a sample of unit area, the spin-dependent eigenstates of Eq. (3) are plane waves,

$$
\psi_{\mathbf{k}, \sigma}(\mathbf{r})=e^{i \mathbf{k} \cdot \mathbf{r}}|\sigma\rangle,
$$

of energy eigenvalues, written in respect with the constant $-\hbar^{2} Q^{2} / 2 m^{*}$

$$
\epsilon_{\mathbf{k}, \sigma}=\frac{\hbar^{2}\left(k_{x}-\sigma Q\right)^{2}}{2 m^{*}}+\frac{\hbar^{2} k_{z}^{2}}{2 m^{*}},
$$

where $\sigma$ is 1 for $|\uparrow\rangle$ and -1 for $|\downarrow\rangle$.

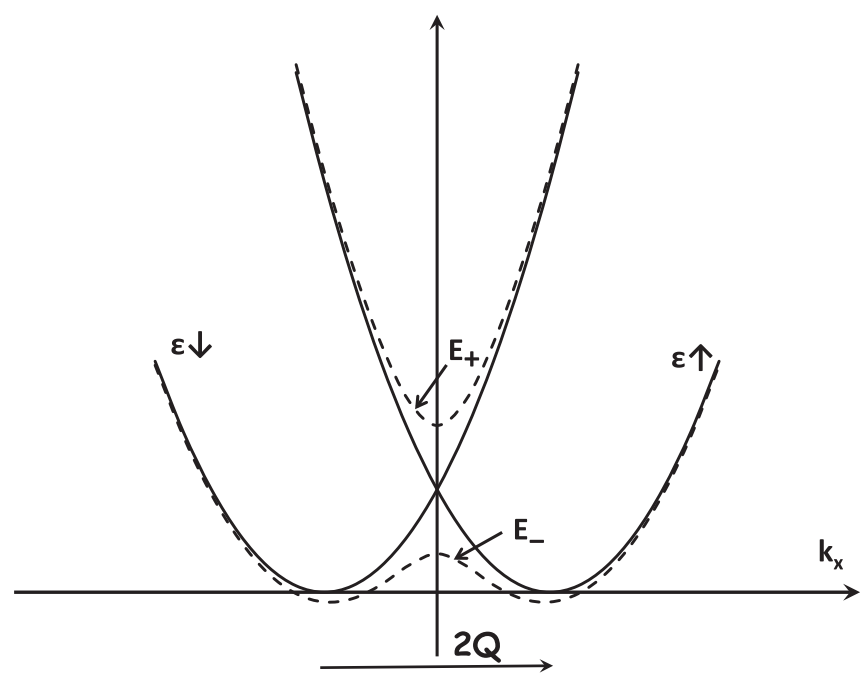

FIG. 1. The single particle spectrum of a $2 \mathrm{D}$ electron system with equal Rashba-Dresselhaus linear couplings. The opposite-spin energies $\varepsilon_{\uparrow}$ and $\varepsilon_{\downarrow}$ are degenerate at $\mathbf{k}=0$, the source of a spin instability that leads to the formation of two new quasiparticles of energies $E_{-}$and $E_{+}$. The states that overlap at $\mathbf{k}=0$ are plane waves of the same momentum.

The itinerant antiferromagnetic order we consider as a basis of our transport theory is a result of the degeneracy in the single particle spectrum at $\mathbf{k}=0$, as described in Fig. 1. The existence of single degeneracy points in the single particle energy spectrum has been known to lead to the establishment of long range magnetic order. Such phases were studied both theoretically and observed experimentally in single quantum wells [18,19], double layers [20-22], and in multilayers [23-25]. Depending on the nature of the single particle states involved in the creation of the spin degeneracy, the resulting magnetic phases where found to be either spiral spin density waves (SDW) [24,25] or canted antiferromagnetic [20,21]. Following the general treatment of spin instabilities in Fermi liquids, first developed by Overhauser in his SDW theory [26,27], one can show that the ground state energy of the system in the presence of the Coulomb interaction, approximated within the Hartree-Fock, is obtained for an antiferromagnetic spin alignment $[15,16]$ which corresponds to two new quasiparticle, whose eigenstates can be written in terms of the variational parameter $\theta_{\mathbf{k}}$ as

$$
\begin{aligned}
& \psi_{\mathbf{k},-}(\mathbf{r})=\left(\cos \theta_{\mathbf{k}}|\uparrow\rangle+\sin \theta_{\mathbf{k}}|\downarrow\rangle\right) e^{i \mathbf{k} \cdot \mathbf{r}}, \\
& \psi_{\mathbf{k},+}(\mathbf{r})=\left(-\sin \theta_{\mathbf{k}}|\uparrow\rangle+\cos \theta_{\mathbf{k}}|\downarrow\rangle\right) e^{i \mathbf{k} \cdot \mathbf{r}} .
\end{aligned}
$$

In the basis of these functions, the single-particle Hamiltonian becomes

$$
H=\left(\begin{array}{cc}
\epsilon_{\mathbf{k}, \uparrow} & -g_{\mathbf{k}} \\
-g_{\mathbf{k}} & \epsilon_{\mathbf{k}, \downarrow}
\end{array}\right),
$$

where $\epsilon_{\mathbf{k}, \sigma}$ are the noninteracting electron energies, while $-g_{\mathbf{k}}$ is the off-diagonal Hartree-Fock coupling potential that describes the generalized exchange between states of opposite spin. Its nonzero value is a direct consequence of the $a b$ initio 
assumption that the ground state of the electron system in the presence of the energy degeneracy at $\mathbf{k}=0$ corresponds to the states described Eq. (6). The eigenvalues generated by solving Eq. (7) are the new single-particle energies,

$$
E_{\mathbf{k}}^{ \pm}=\frac{1}{2}\left[\epsilon_{\mathbf{k}, \downarrow}+\epsilon_{\mathbf{k}, \uparrow} \pm \sqrt{\left(\epsilon_{\mathbf{k}, \downarrow}-\epsilon_{\mathbf{k}, \uparrow}\right)^{2}+g_{\mathbf{k}}^{2}}\right]
$$

expressions that pinpoint $g_{\mathbf{k}}$ as the gap between the singleparticle energy states at the point of degeneracy $\mathbf{k}=0$. They also determine the energy dependence of the occupation numbers, self-consistently, $f_{\mathbf{k} \pm}=\left(e^{\left(E_{\mathbf{k} \pm}-E_{F}\right) / k_{B} T}+1\right)^{-1}$. At minimum total energy, the variational parameter $\theta_{\mathbf{k}}$ which determines the direction of the single particle spin polarization satisfies

$$
\tan 2 \theta_{\mathbf{k}}=\frac{g_{\mathbf{k}}}{\epsilon_{\mathbf{k}, \downarrow}-\epsilon_{\mathbf{k}, \uparrow}},
$$

leading to the self-consistent equation for the gap function $g_{\mathbf{k}}$,

$$
g_{\mathbf{k}}=\sum_{\mathbf{k}^{\prime}} v\left(\mathbf{k}-\mathbf{k}^{\prime}\right) \frac{g_{\mathbf{k}^{\prime}}\left(f_{\mathbf{k}^{\prime}-}-f_{\mathbf{k}^{\prime}+}\right)}{\sqrt{\left(\epsilon_{\mathbf{k}^{\prime}, \downarrow}-\epsilon_{\mathbf{k}^{\prime}, \uparrow}\right)^{2}+g_{\mathbf{k}^{\prime}}^{2}}},
$$

with $v\left(\mathbf{k}-\mathbf{k}^{\prime}\right)=2 \pi e^{2} / \kappa\left|\mathbf{k}-\mathbf{k}^{\prime}\right|$ the Fourier transform of the bare Coulomb interaction in a two-dimensional (2D) environment of dielectric constant $\kappa$. The Fermi energy $E_{F}$ is established by the constraint that the number of particles in the system remains the same,

$$
n=\sum_{\mathbf{k}, \pm} f_{\mathbf{k} \pm}
$$

The average of the spin operator on these states generates a spin polarization only along the $\hat{x}$ axis, parallel to the displacement vector $\mathbf{Q}$,

$$
\mathbf{P}=\sum_{\mathbf{k}, \pm}\left\langle\psi_{\mathbf{k} \pm}|\sigma| \psi_{\mathbf{k} \pm}\right\rangle f_{\mathbf{k} \pm}=\hat{x} \sum_{\mathbf{k}} \sin 2 \theta_{\mathbf{k}}\left(f_{\mathbf{k}-}-f_{\mathbf{k}+}\right) .
$$

\section{TRANSPORT IN THE ANTIFERROMAGNETIC STATE}

We model the transport properties of the antiferromagnetic state as a two-channel process occurring separately in the + and - minibands. Since the single particle states described by the canonical transformations Eq. (6) represent exact eigenstates of the noninteracting Hamiltonian, the spin and charge currents carried by these states are defined as the averages of the generalized momentum $\mathbf{p}-\hbar \mathbf{Q} \sigma_{z}$ on these states, multiplied correspondingly by the electric charge and spin unit. Therefore, the total charge $(c)$ and $\hat{x}$-polarized spin $(s)$ currents are given by

$$
\begin{gathered}
\mathbf{j}_{c}=-e \sum_{\mathbf{k}, \pm}\left\langle\psi^{ \pm}\left|\frac{\mathbf{p}-\hbar \mathbf{Q} \sigma_{z}}{m^{*}}\right| \psi^{ \pm}\right\rangle \Delta f_{\mathbf{k} \pm} \\
\mathbf{j}_{s}^{x}=\frac{\hbar}{2} \sum_{\mathbf{k}, \sigma, \pm}\left\langle\psi^{ \pm}\left|\frac{\left(\mathbf{p}-\hbar \mathbf{Q} \sigma_{z}\right) \sigma_{x}+\sigma_{x}\left(\mathbf{p}-\sigma_{z} \hbar \mathbf{Q}\right)}{2 m^{*}}\right| \psi^{ \pm}\right\rangle \Delta f_{\mathbf{k} \pm}
\end{gathered}
$$

where $\Delta f_{\mathbf{k} \pm}$ is the deviation from equilibrium of the single particle occupation number under the effect of the external perturbation.
In the following considerations we will focus only on the deviation in the presence of an electric field rather than a temperature gradient, as the Seebeck coefficient in both the charge and spin case is calculated by using the Mott formula which correlates the Seebeck coefficient with the derivative of the logarithm of the corresponding differential conductivity $\sigma_{c, s}(E)$ evaluated at the Fermi energy. This algorithm is similarly applicable for both spin and charge are carried by single particle states that satisfy the same transport equation as discussed in Ref. [7]:

$$
S_{c, s}=-\left.\frac{\pi}{3} \frac{k_{B}}{e} \frac{d \ln \sigma_{c, s}(E)}{d E}\right|_{E=E_{F}} .
$$

In the presence of an electric field $\mathcal{E}$, the out-of-equilibrium part of the distribution function is written in the relaxation time approximation as

$$
\Delta f_{\mathbf{k} \pm}=-e\left(\mathcal{E} \cdot \mathbf{v}_{\mathbf{k} \pm}\right) \tau\left(E_{\mathbf{k} \pm}\right)\left(-\frac{d f_{\mathbf{k} \pm}}{d E_{\mathbf{k} \pm}}\right) .
$$

$\tau\left(E_{\mathbf{k} \pm}\right)$ is the relaxation time, considered a function of energy only.

Considering the temperature range of interest in this problem, henceforth we approximate $\left(-d f_{\mathbf{k} \pm} / d E_{\mathbf{k} \pm}\right)=\delta\left(E_{F}-\right.$ $\left.E_{\mathbf{k} \pm}\right)$. Moreover, in the same temperature range, we assume that the variation of the antiferromagnetic gap is negligible and $g_{k}(T)=$ const.

We define the quasiparticle velocities,

$$
\begin{aligned}
\mathbf{v}_{\mathbf{k} \pm} & =\left\langle\psi^{ \pm}\left|\frac{\mathbf{p}-\hbar \mathbf{Q} \sigma_{z}}{m^{*}}\right| \psi^{ \pm}\right\rangle=\frac{1}{\hbar} \nabla_{\mathbf{k}} E_{\mathbf{k} \pm} \\
& =\frac{\hbar}{m^{*}}\left(\mathbf{k} \pm \mathbf{Q} \cos 2 \theta_{\mathbf{k}}\right),
\end{aligned}
$$

and note that depending on the relative orientation of the electric field $\mathcal{E}$ and the wave vector $\mathbf{Q}$ there are two different transport modes, normal $(n)$ and parallel $(p)$,

$$
\mathbf{v}_{\mathbf{k} \pm}^{(n)}=\hbar \mathbf{k} / m^{*}, \quad \mathbf{v}_{\mathbf{k} \pm}^{(p)}=\frac{\hbar \mathbf{Q}}{m^{*} Q^{2}}\left(\mathbf{k} \cdot \mathbf{Q} \pm \mathbf{Q}^{2} \cos 2 \theta_{\mathbf{k}}\right) .
$$

The corresponding $n$ or $p$ differential conductivity expressions for the charge and spin current, polarized along the $\hat{x}$ direction result immediately,

$$
\begin{aligned}
\sigma^{(n, p)}(E)= & e^{2} \sum_{\mathbf{k}, \pm} \frac{\hbar \mathbf{k}}{m^{*}} \mathbf{v}_{\mathbf{k} \pm}^{(n, p)} \tau^{(n, p)}\left(E_{\mathbf{k} \pm}\right) \delta\left(E-E_{\mathbf{k} \pm}\right) \\
\sigma_{s}^{x(n, p)}(E)= & -\frac{e \hbar}{2} \sum_{\mathbf{k}, \pm} \operatorname{sgn}( \pm) \sin 2 \theta_{\mathbf{k}} \frac{\hbar \mathbf{k}}{m^{*}} \mathbf{v}_{\mathbf{k} \pm}^{(n, p)} \tau^{(n, p)}\left(E_{\mathbf{k} \pm}\right) \\
& \times \delta\left(E-E_{\mathbf{k} \pm}\right)
\end{aligned}
$$

$[\operatorname{sgn}( \pm)= \pm 1]$

The weak variation of the differential charge and spin conductivities, Eq. (19) with energy generates the very small Seebeck coefficient in homogeneous systems. Transport coefficients can be enhanced through scattering processes that preserve the population imbalance of up and down spin states created through antiferromagnetic order by introducing an energy-dependent relaxation lifetime. As shown in Ref. [14] this can be realized by considering a scattering potential that 
has a magnetic component, of the type,

$$
\sum_{i}\left[V \delta\left(\mathbf{r}-\mathbf{R}_{i}\right)+J \sigma \cdot \mathbf{S}_{i} \delta\left(\mathbf{r}-\mathbf{R}_{i}\right)\right]
$$

where $\sigma$ is the electron spin at the site of the magnetic scatterer of spin $\mathbf{S}$. $V$ is the isotropic scattering potential and $J$ is the strength of the magnetic potential. For this choice of potential, a long but straightforward calculation based on applying the Fermi golden rule inside the collision integral of the Boltzmann transport equation provides values for the normal and parallel relaxation times [14]. Thus,

$$
\begin{aligned}
\frac{1}{\tau^{(n)}\left(E_{\mathbf{k} \pm}\right)}= & \frac{\pi N_{i}}{\hbar^{2}}\left\{\left(V^{2}+J^{2} S_{z}^{2}\right) I_{0}\left(E_{\mathbf{k} \pm}\right)\right. \\
& +\sin 2 \theta_{\mathbf{k}}\left(V^{2}-J^{2} S_{z}^{2}\right) I_{1}\left(E_{\mathbf{k} \pm}\right) \\
& +\frac{\left(J S^{-}\right)^{2}}{2} \eta(E, \Delta E)\left[I_{0}\left(E_{\mathbf{k} \pm}-\Delta E\right)\right. \\
& \left.+\sin 2 \theta_{\mathbf{k}} I_{1}\left(E_{\mathbf{k} \pm}-\Delta E\right)\right] \\
& +\frac{\left(J S^{+}\right)^{2}}{2} \eta\left(E_{\mathbf{k} \pm},-\Delta E\right)\left[I_{0}\left(E_{\mathbf{k} \pm}+\Delta E\right)\right. \\
& \left.\left.+\sin 2 \theta_{\mathbf{k}} I_{1}\left(E_{\mathbf{k} \pm}+\Delta E\right)\right]\right\} .
\end{aligned}
$$

This result assumes a concentration $N_{i}$ of impurities with same spin $\mathbf{S}$ whose projection on the $\hat{z}$ axis is $S_{z}$, while its perpendicular components are $\left(S^{ \pm}\right)^{2}=S(S+1)-S_{z}^{2}$. In the numerical evaluations $S_{z}$ is replaced by its thermal average. $\Delta E$ is the Zeeman splitting in the presence of the magnetic impurity which parametrizes the function $\eta\left(E_{\mathbf{k} \pm}, \Delta E\right)$, a measure of the collision inelasticity given by

$$
\eta\left(E_{\mathbf{k} \pm}, \Delta E\right)=\frac{e^{\left(E_{\mathbf{k} \pm}-E_{F}\right) / k_{B} T}+1}{e^{\left(E_{\mathbf{k} \pm}-E_{F}\right) k_{B} T}+e^{-\Delta E / k_{B} T}}
$$

$I_{0}\left(E_{\mathbf{k} \pm}\right)$ and $I_{1}\left(E_{\mathbf{k} \pm}\right)$ represent the density of states and the spin polarization at energy $E_{\mathbf{k} \pm}$,

$$
\begin{aligned}
& I_{0}\left(E_{\mathbf{k} \pm}\right)=\sum_{\mathbf{k}^{\prime}} \delta\left(E_{\mathbf{k} \pm}-E_{\mathbf{k}^{\prime} \pm}\right), \\
& I_{1}\left(E_{\mathbf{k} \pm}\right)=\sum_{\mathbf{k}^{\prime}} \sin 2 \theta_{\mathbf{k}^{\prime}} \delta\left(E_{\mathbf{k} \pm}-E_{\mathbf{k}^{\prime} \pm}\right) .
\end{aligned}
$$

The parallel mode relaxation time is

$$
\tau^{(p)}\left(E_{\mathbf{k} \pm}\right)=\tau^{(n)}\left(E_{\mathbf{k} \pm}\right)\left[1+\frac{\pi N_{i}}{\hbar} \frac{\cos 2 \theta_{n}\left(E_{\mathbf{k} \pm}\right)\left(V^{2}-J^{2} S_{z}^{2}\right)}{v_{\mathbf{k} \pm}^{p}} \frac{\sum_{\mathbf{k}^{\prime}} \cos 2 \theta_{\mathbf{k}^{\prime}} v_{\mathbf{k}^{\prime} \pm}^{p} \tau^{(n)}\left(E_{\mathbf{k} \pm}\right) \delta\left(E_{\mathbf{k} \pm}-E_{\mathbf{k}^{\prime} \pm}\right)}{1-\frac{\pi N_{i}}{\hbar}\left(V^{2}-J^{2} S_{z}^{2}\right) \sum_{\mathbf{k}^{\prime}} \cos ^{2} 2 \theta_{\mathbf{k}^{\prime}} \tau^{(n)}\left(E_{\mathbf{k} \pm}\right) \delta\left(E_{\mathbf{k} \pm}-E_{\mathbf{k}^{\prime} \pm}\right)}\right] .
$$

\section{RESULTS AND CONCLUSION}

We present numerical results of the gap equation for a GaAs (effective mass $m^{*}=0.067$, dielectric constant $\kappa=13$ ) quantum well of electron density $n=4 \times 10^{15} \mathrm{~m}^{-2}$. The $\alpha=\beta$ state was identified from the antilocalization peak of the quantum corrections to the conductivity in the weak scattering regime [28] and found to correspond to a value $\alpha=9 \times 10^{2}$ $\mathrm{m} / \mathrm{s}$. For the given particle concentration, we define the Fermi momentum of the isotropic system (in the absence of SOI), $k_{F}=\sqrt{2 \pi n}=1.58 \times 10^{8} \mathrm{~m}^{-1}$ which henceforth will be used as a unit in the momentum space. Correspondingly, the energy scale of the problem is set by the single particle Fermi energy $E_{F}=\hbar^{2} k_{F}^{2} / 2 m^{*}=12.88 \mathrm{meV}$. For these parameters, $Q=2 m^{*} \alpha / \hbar=2 \alpha / v_{F}=7.2 \times 10^{-3} k_{F}$, while the Fermi temperature which sets the temperature scale is $T_{F}=150 \mathrm{~K}$.

An iterative calculation is first employed to obtain a solution of the gap equation plotted as a function of temperature in Fig. 2. To incorporates a qualitative description of the additional screening that can appear in the system on account of short range Coulomb interaction effects beyond the Hartree-Fock approximation, our computation is performed with an Yukawa potential of screening constant $\mu=10^{-3}$ (expressed throughout in $k_{F}$ units), whose Fourier transform is $v(q)=2 \pi e^{2} / \kappa \sqrt{q^{2}+\mu^{2}}$. This step is necessary to avoid the divergence of the Fourier transform in one dimension in the numerical treatment. At each temperature, the Fermi level $E_{F}$ is calculated from the condition that the particle density remains constant, Eq. (11). Here we show the variation of the gap function at the center of the Fermi disk, a good indicator for the temperature range in which one expects to have an antiferromagnetic state. Our results indicate that the critical temperature of the system is about $90 \mathrm{~K}$. In the following

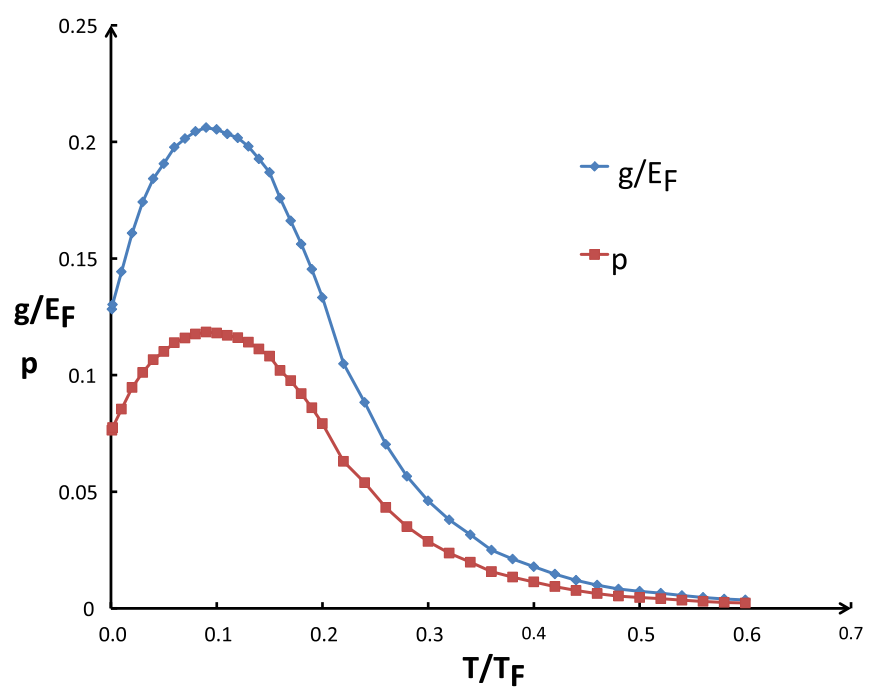

FIG. 2. The variation of the gap function at the center of the Fermi surface and of the spin polarization for a screening constants $\mu_{1}=10^{-3}$ in a GaAs quantum well with $n=4 \times 10^{15} \mathrm{~m}^{-2}$, $Q=7.2 \times 10^{-3} k_{F}$ as a function of temperature. 


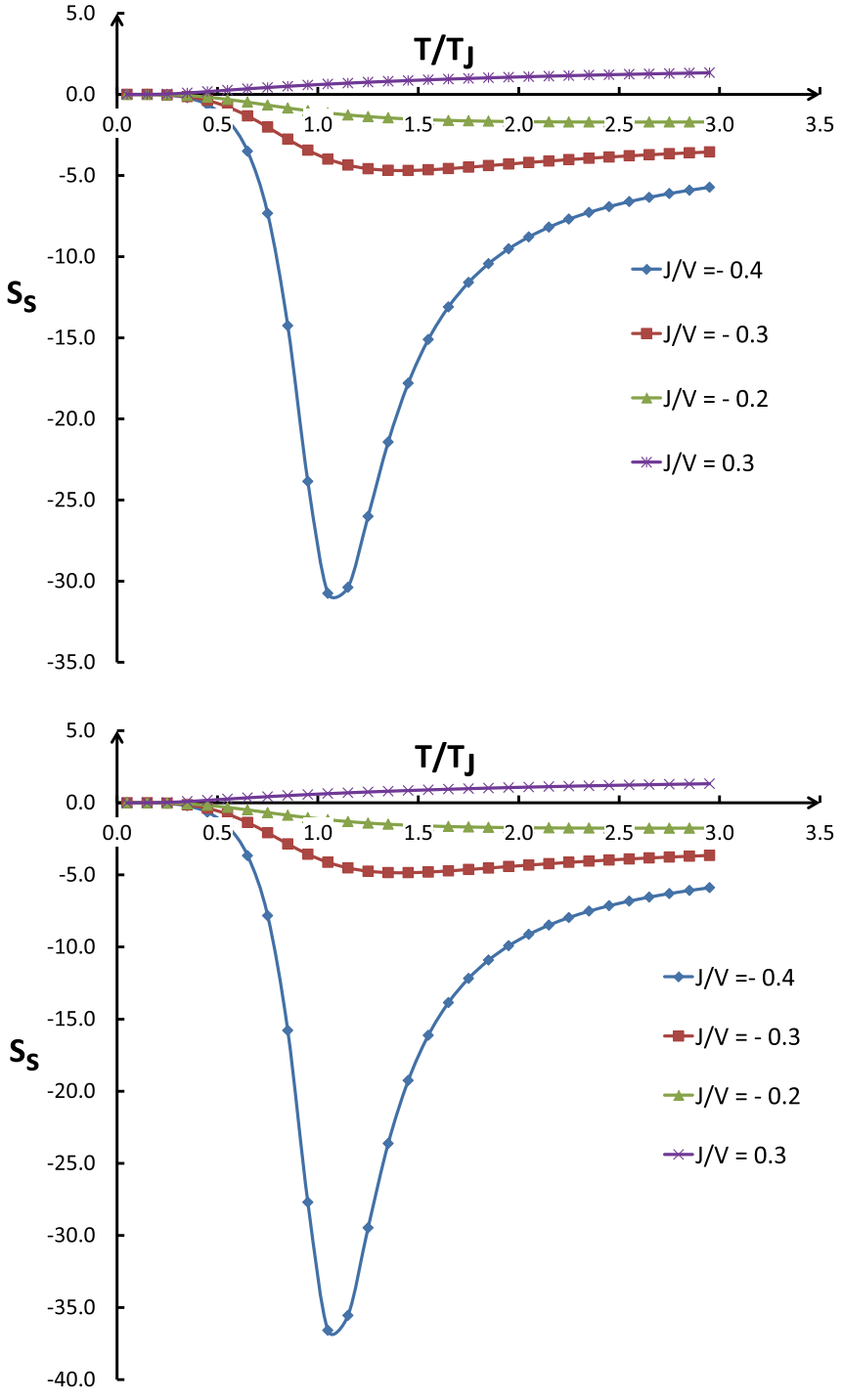

FIG. 3. The variation of the spin Seebeck coefficient, expressed in $k_{B} / e=86.3 \mu \mathrm{V} / \mathrm{K}$ units, for the normal in (a) and parallel in (b) mode with temperature for different values of the ratio $J / V$. The gap value is $0.2 E_{F}$, while the polarization is $10 \%$. The temperature unit is set by the spin-flip energy and corresponds to $35 \mathrm{~K}$. evaluations the ratio of the spin-dependent scattering potential $J$ to the isotropic potential $V, J / V$, is used as a parameter. The former is set to $J=0.03 \mathrm{eV}$, while $V$ is allowed to vary. The impurity spin is $S=5 / 2$. The chosen values of $J$ and $p$ give the value of the Zeeman splitting $\Delta E=p J$ and establish the temperature unit $\Delta E / k_{B} . T=1$ corresponds to $35 \mathrm{~K}$. Since the temperature unit imposed by the magnetic impurity scattering is much smaller than the critical temperature of the antiferromagnetic transition, we will assume that in the low temperature range we are interested in the gap variation with temperature is negligible and consequently the gap function is considered constant, equal to $0.2 E_{F}$.

The small value of the $Q / k_{F}$ in the GaAs quantum well selected for the numerical evaluation leads to a charge Seebeck coefficient, which depends on $\left(Q / k_{F}\right)^{2}$, that is very close in value to that of a homogeneous system and we choose not to plot it. In contrast, as the two minibands have opposite spin polarization, the spin Seenbeck coefficient, linear in $Q / k_{F}$, is finite, while its amplitude increases with the strength of the magnetic impurity scattering.

In Fig. 3 we present the values of the spin Seebeck coefficient for different values of the ratio $J / V$ for the normal and parallel transport modes. The current of course is spin polarized after $\hat{x}$. While for $J>0$ the spin Seebeck coefficient value increases weakly with the strength of the magnetic scattering vs the isotropic magnetic scattering, its magnitude varies dramatically for $J<0$, where an anomalous high value is registered for a sufficiently large magnetic scattering. The temperature at which the peak in the spin Seebeck coefficient value occurs is determined by the Zeeman energy corresponding to a spin-flip of the magnetic impurity.

In conclusion, we showed that in an 2D electron system with equal Rashba and Dresselhaus spin-orbit couplings, an unusual high value of the Seebeck coefficient is possible to exist at low temperature when the impurity scattering contains a magnetic component. This result originates in the confluence of the magnetic order imposed by the minimization of the total energy of the electron system in the presence of the Coulomb interaction and the unidirectional energy transfer in the spinflip scattering. The overall amplitude of the effect is determined by the degree of polarization of the electron system and the strength of the magnetic scattering.
[1] J. Schliemann, J. C. Egues, and D. Loss, Phys. Rev. Lett. 90, 146801 (2003).

[2] J. D. Koralek, C. P. Weber, J. Orenstein, B. A. Bernevig, S.-C. Zhang, S. Mack, and D. D. Awschalom, Nature (London) 458, 610 (2009).

[3] M. P. Walser, W. Reichl, C. Wegscheider, and G. Salis, Nat. Phys. 8, 757 (2012).

[4] M. Kohda, V. Lechner, Y. Kunihashi, T. Dollinger, P. Olbrich, C. Schönhuber, I. Caspers, V. V. Bel'kov, L. E. Golub, D. Weiss, K. Richter, J. Nitta, and S. D. Ganichev, Phys. Rev. B 86, 081306 (2012).

[5] F. G. Pikus and G. E. Pikus, Phys. Rev. B 51, 16928 (1995).
[6] G. E. Bauer, A. H. MacDonald, and S. Maekawa, Solid State Commun. 150, 459 (2010).

[7] G. E. W. Bauer, E. Saitoh, and B. J. van Wees, Nat. Mater. 11, 391 (2012).

[8] K. Uchida, S. Takahashi, K. Harii, J. Ieda, W. Koshibae, K. Ando, S. Maekawa, and E. Saitoh, Nature (London) 455, 778 (2008).

[9] K. Uchida, T. Ota, K. Harii, S. Takahashi, S. Maekawa, Y. Fujikawa, and E. Saitoh, Solid State Commun. 150, 524 (2010).

[10] C. M. Jaworski, J. Yang, S. Mack, D. D. Awschalom, J. P. Heremans, and R. C. Myers, Nat. Mater. 9, 898 (2010).

[11] C. M. Jaworski, R. C. Myers, E. Johnston-Halperin, and J. P. Heremans, Nature (London) 487, 210 (2012). 
[12] J.-i. Ohe, H. Adachi, S. Takahashi, and S. Maekawa, Phys. Rev. B 83, 115118 (2011).

[13] K. Uchida, J. Xiao, H. Adachi, J. Ohe, S. Takahashi, J. Leda, T. Ota, Y. Kajiwara, U. Umezawa, H. Kawai, G. Bauer, S. Maekawa, and E. Saitoh, Nat. Mater. 9, 894 (2010).

[14] L. L. Van Zandt and A. W. Overhauser, Phys. Rev. 141, 583 (1966).

[15] D. C. Marinescu, Physica E 69, 34 (2015).

[16] J. Capps, D. C. Marinescu, and A. Manolescu, Phys. Rev. B 91, 165301 (2015).

[17] M. I. Alomar, L. Serra, and D. Sánchez, Phys. Rev. B 91, 075418 (2015).

[18] G. F. Giuliani and J. J. Quinn, Phys. Rev. B 31, 6228 (1985).

[19] F. F. Fang and P. J. Stiles, Phys. Rev. 174, 823 (1968).
[20] L. Zheng, R. J. Radtke, and S. Das Sarma, Phys. Rev. Lett. 78, 2453 (1997).

[21] S. Das Sarma, S. Sachdev, and L. Zheng, Phys. Rev. B 58, 4672 (1998).

[22] V. Pellegrini, A. Pinczuk, B. S. Dennis, A. S. Plaut, L. N. Pfeiffer, and K. W. West, Phys. Rev. Lett. 78, 310 (1997).

[23] L. Brey, Phys. Rev. Lett. 81, 4692 (1998).

[24] D. C. Marinescu, J. J. Quinn, and G. F. Giuliani, Phys. Rev. B 61, 7245 (2000).

[25] L. Zheng, D. C. Marinescu, and G. F. Giuliani, Phys. Rev. B 84, 205321 (2011).

[26] A. W. Overhauser, Phys. Rev. Lett. 4, 462 (1960).

[27] A. W. Overhauser, Phys. Rev. 128, 1437 (1962).

[28] D. Zumbühl (private communication, 2014). 